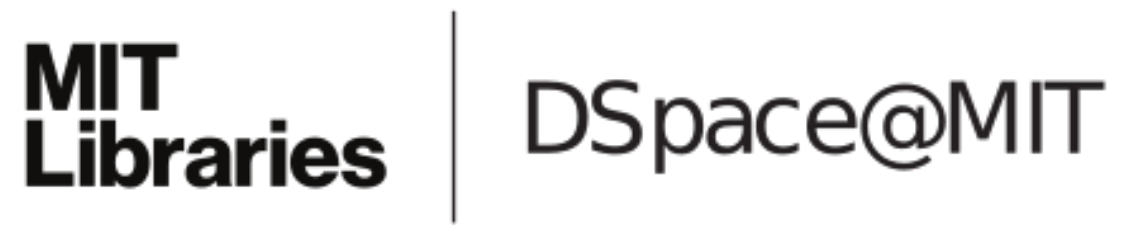

\author{
MIT Open Access Articles
}

Mechanism for enhanced oxygen reduction kinetics at the (La,Sr)CoO3-\#/(La,Sr)2CoO4+\# hetero-interface

The MIT Faculty has made this article openly available. Please share how this access benefits you. Your story matters.

Citation: Han, Jeong Woo, and Bilge Yildiz. “Mechanism for Enhanced Oxygen Reduction Kinetics at the (La,Sr)CoO3- $\delta /(L a, S r) 2 C o 04+\delta$ Hetero-Interface." Energy \& Environmental Science 5, no. 9 (2012): 8598.

As Published: http://dx.doi.org/10.1039/c2ee03592h

Publisher: Royal Society of Chemistry

Persistent URL: http://hdl.handle.net/1721.1/86188

Version: Author's final manuscript: final author's manuscript post peer review, without publisher's formatting or copy editing

Terms of use: Creative Commons Attribution-Noncommercial-Share Alike 


\title{
Mechanism for enhanced oxygen reduction kinetics at the $(\mathrm{La}, \mathrm{Sr}) \mathrm{CoO}_{3-\delta} /(\mathrm{La}, \mathrm{Sr})_{2} \mathrm{CoO}_{4+\delta}$ hetero-interface
}

\author{
Jeong Woo Han and Bilge Yildiz* \\ Laboratory for Electrochemical Interfaces, Department of Nuclear Science and Engineering, \\ Massachusetts Institute of Technology, \\ 77 Massachusetts Avenue, Cambridge, Massachusetts 02139, USA
}

\begin{abstract}
The recently reported fast oxygen reduction kinetics at the interface of $(\mathrm{La}, \mathrm{Sr}) \mathrm{CoO}_{3-\delta}\left(\mathrm{LSC}_{113}\right)$ and $(\mathrm{La}, \mathrm{Sr})_{2} \mathrm{CoO}_{4+\delta}\left(\mathrm{LSC}_{214}\right)$ phases opened up new questions for the potential role of dissimilar interfaces in advanced cathodes for solid oxide fuel cells (SOFCs). Using first-principles based calculations in the framework of density functional theory, we quantitatively probed the possible mechanisms that govern the oxygen reduction activity enhancement at this hetero-interface as a model system. Our findings show that both the strongly anisotropic oxygen incorporation kinetics on the $\mathrm{LSC}_{214}$ and the lattice strain in the vicinity of the interface are important contributors to such enhancement. The $\mathrm{LSC}_{214}(100)$ surface exposed to the ambient at the $\mathrm{LSC}_{113} / \mathrm{LSC}_{214}$ interface facilitates oxygen incorporation because the oxygen molecules very favorably adsorb on it compared to the $\operatorname{LSC}_{214}(001)$ and $\operatorname{LSC}_{113}(001)$ surfaces, providing a large source term for oxygen incorporation. Lattice strain field present near the hetero-interface accelerates oxygen incorporation kinetics especially on $\operatorname{LSC}_{113}(001)$. At $500{ }^{\circ} \mathrm{C} 4 \times 10^{2}$ times faster oxygen incorporation kinetics is predicted in the vicinity of the $\mathrm{LSC}_{113} / \mathrm{LSC}_{214}$ heterointerface with $50 \%$ Sr-doped LSC $_{214}$ compared to that on the single phase LSC $_{113}(001)$ surface. Contributions from both the anisotropy and local strain effects are of comparable magnitude. The insights obtained in this work suggest that hetero-structures which have a large area of (100) surfaces and smaller thickness in [001] direction of the Ruddlesden-Popper phases, and larger tensile strain near the interface would be promising for high-performance cathodes.
\end{abstract}

Keywords: solid oxide fuel cell, oxygen reduction reaction, cathode, anisotropy, strain, perovskite, Ruddlesden-Popper, $(\mathrm{La}, \mathrm{Sr}) \mathrm{CoO}_{3},(\mathrm{La}, \mathrm{Sr})_{2} \mathrm{CoO}_{4+\delta}$, density functional theory

* Corresponding author. Email: byildiz@mit.edu 


\section{Graphical Abstract}
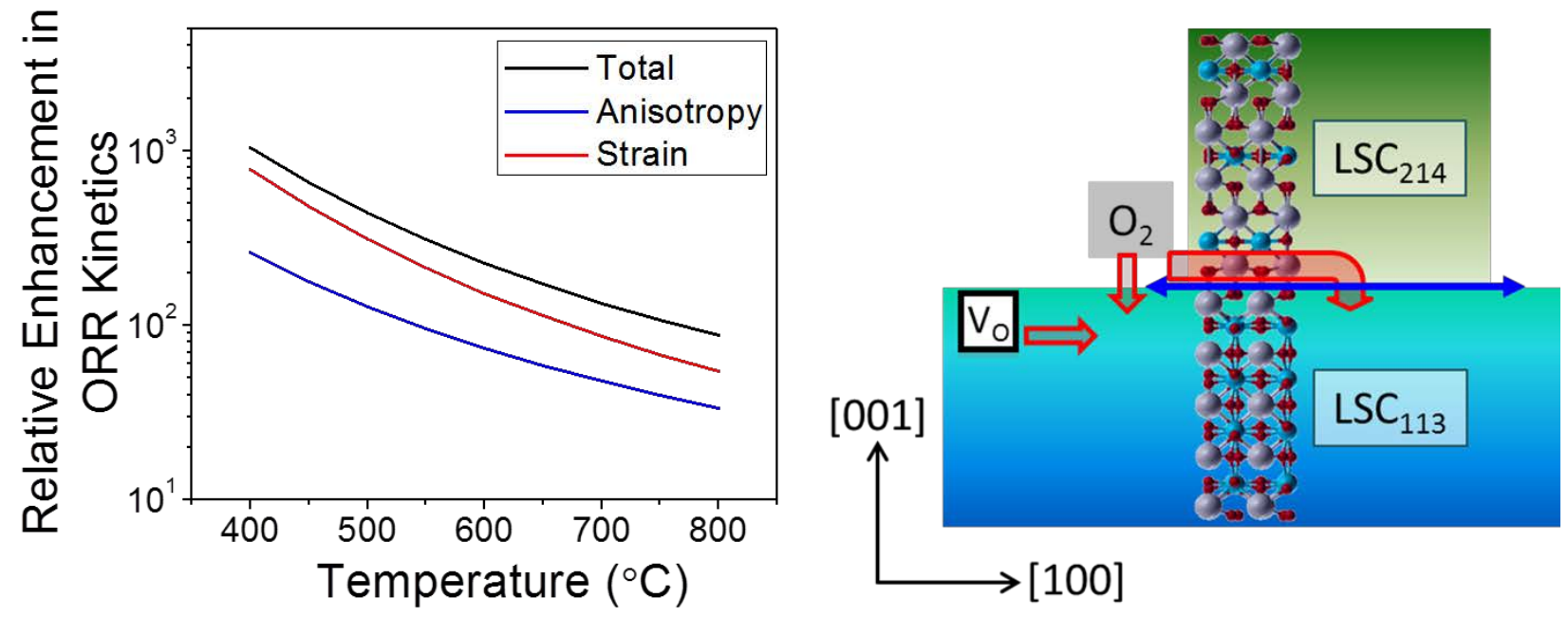

Anisotropic oxygen incorporation on $(\mathrm{La}, \mathrm{Sr})_{2} \mathrm{CoO}_{4+\delta}$ and the lattice strain near the (La, $\left.\mathrm{Sr}\right) \mathrm{CoO}_{3-\delta}$ $/(\mathrm{La}, \mathrm{Sr})_{2} \mathrm{CoO}_{4+\delta}$ interface serve as two possible sources to accelerate the oxygen reduction kinetics on the hetero-structure by $4 \times 10^{2}$ times at $500{ }^{\circ} \mathrm{C}$.

\section{Broader context}

High efficiency and fuel flexibility of Solid Oxide Fuel Cells (SOFCs) render them attractive as a sustainable energy conversion technology. There is growing interest in the design of dissimilar interfaces at the nano-scale to enable high-performance SOFC cathodes with fast oxygen reduction reaction (ORR) kinetics at lower temperatures than their traditional operation temperature of $800{ }^{\circ} \mathrm{C}$. A motivating example for this purpose has been the hetero-structure made of the (La,Sr) $\mathrm{CoO}_{3-\delta}$ and (La,Sr) ${ }_{2} \mathrm{CoO}_{4+\delta}$ phases with highly active interfaces. Two previous experimental reports have observed $10^{3}-10^{4}$ times enhancement in ORR kinetics at 500$550{ }^{\circ} \mathrm{C}$ arising from the interface of these two phases. However, these empirical observations have not yet reached a fundamental understanding of why these interfaces are so highly active to ORR. Here we use first principles based calculations to quantitatively demonstrate two important mechanisms that can govern the unusually high ORR activity of this hetero-interface system; the strongly anisotropic oxygen adsorption and incorporation kinetics on the ( $\mathrm{La}, \mathrm{Sr})_{2} \mathrm{CoO}_{4}(100)$ and the lattice strain effect near the interface. Our findings provide new insights for designing novel interfaces in high-performance cathodes. Furthermore, these results are applicable broadly to 
other systems were oxide interfaces are critical in charge transfer kinetics, as batteries, catalysis, and sensors.

\section{Introduction}

The slow rate of oxygen reduction rate at the cathode has been the primary limitation to the performance of solid oxide fuel cells (SOFCs) at intermediate temperatures $\left(500-700{ }^{\circ} \mathrm{C}\right)^{1-5}$ that is important for materials stability and system cost. ${ }^{1,3,5,6}$ This has led to an extensive search for highly active cathode materials that accelerate the kinetics of oxygen reduction reaction (ORR). Traditionally, perovskite type transition metal oxides $\left(\mathrm{ABO}_{3}\right)$ have been widely investigated as SOFC cathodes. 2, 3, 7-10 More recent studies highlight the potential of layered materials, such as Ruddlesden-Popper (RP) family of $\mathrm{A}_{n+1} \mathrm{~B}_{n} \mathrm{O}_{3 n+1}$, as desirable cathodes due to their fast and anisotropic oxygen incorporation and transport properties. ${ }^{6,11-14}$ Interestingly, Sase et al. demonstrated from Secondary Ion Mass Spectrometry (SIMS) that the oxygen surface exchange rate at the hetero-interface of $\mathrm{La}_{0.6} \mathrm{Sr}_{0.4} \mathrm{CoO}_{3} /\left(\mathrm{La}_{0.5} \mathrm{Sr}_{0.5}\right)_{2} \mathrm{CoO}_{4}$ thin films is enhanced by about $10^{3}$ times at $500{ }^{\circ} \mathrm{C}$ compared to that on the single-phase $\mathrm{La}_{0.6} \mathrm{Sr}_{0.4} \mathrm{CoO}_{3}$ surfaces. ${ }^{15}$ Following up this study, Crumlin et al., using electrochemical impedance spectroscopy measurements, reported also an ORR activity enhancement up to about $10^{3}-10^{4}$ times at $550{ }^{\circ} \mathrm{C}$ on the thin film $\mathrm{La}_{0.8} \mathrm{Sr}_{0.2} \mathrm{CoO}_{3-\delta}$ cathodes whose surfaces are decorated with $\left(\mathrm{La}_{0.5} \mathrm{Sr}_{0.5}\right)_{2} \mathrm{CoO}_{4}$ islands (an thus, with a high density of $\mathrm{LSC}_{113} / \mathrm{LSC}_{214}$ interfaces). ${ }^{16}$ While it is evident that the interfacial regions are contributing to this unusually high ORR activity, the governing mechanism behind these empirical observations was not identified to date. Obtaining a microscopic level understanding for such behavior is important for designing novel interfaces for very high-performance SOFC cathodes. In this paper, we hypothesize two possible mechanisms for the ORR enhancement on this hetero-structure; the first is the anisotropically fast $\mathrm{O}_{2}$ incorporation into the $\operatorname{LSC}_{214}(100)$ surface present at this interface (as is the configuration reported in both ref. 15 and 16), and the second is the effect of lattice strain on the oxygen reduction reactions near the hetero-interface.

In support of our first hypothesis, several compositions belonging to the first member of the RP series, $\mathrm{A}_{2} \mathrm{BO}_{4}$, were shown to exhibit fast anisotropic oxygen exchange and diffusion kinetics. ${ }^{6,12,13,17-23}$ For example, on $\mathrm{La}_{2} \mathrm{NiO}_{4+\delta}$ and $\mathrm{La}_{2-x} \mathrm{Sr}_{x} \mathrm{CuO}_{4-\delta}, 10^{2}-10^{3}$ times larger oxygen interstitial diffusion and surface exchange coefficients along the $a b$ plane ((001) plane) than on 
the $c$ plane ((100) plane) were reported. ${ }^{17,19,21,22}$ Similar reports for (La,Sr) ${ }_{2} \mathrm{CoO}_{4+\delta}$ are scarce to date. We have previously shown a fast anisotropic oxygen interstitial transport along the $a b$ plane in $\mathrm{La}_{2} \mathrm{CoO}_{4+\delta}$ computationally. ${ }^{13}$ There are no reports in literature yet on its surface exchange kinetics on each major plane. $\mathrm{LSC}_{214}(100)$ surface can play an important role as an oxygen source boundary condition by an energetically favorable $\mathrm{O}_{2}$ adsorption in enabling fast ORR kinetics at the $\mathrm{LSC}_{113} / \mathrm{LSC}_{214}$ interface, In fact, this surface is exposed to air in both Sase et al.'s work ${ }^{15}$ and as well as in Crumlin et al.'s work ${ }^{16}$ since they both deposited the LSC $_{214}$ on top of $\mathrm{LSC}_{113}$ in creating a highly textured hetero-interface.

In support of our second hypothesis, lattice strain was recently shown to alter the oxygen defect chemistry as well as the oxygen reaction and diffusion kinetics on perovskite oxides. Strain fields are often induced by the lattice mismatch near a dissimilar interface. As shown in Fig. 1, the (La,Sr) $\mathrm{CoO}_{3} /(\mathrm{La}, \mathrm{Sr})_{2} \mathrm{CoO}_{4}$ layered structure (constructed using density functional theory (DFT), see Sec. 2) imposes a $+1.9 \%$ planar strain in (001) plane of $\mathrm{LSC}_{113}$, and a $-0.8 \%$ planar strain in (001) plane of $\mathrm{LSC}_{214}$. These theoretical values are expected to give an upper bound of the magnitude of strain near the $\mathrm{LSC}_{113} / \mathrm{LSC}_{214}$ hetero-interface. Lattice strain is shown (by works including that of the authors here) to have a significant impact on facilitating oxygen ion transport, ${ }^{24-28}$ vacancy formation, ${ }^{29-32}$ and surface adsorption ${ }^{27,} 30$ - specific to this paper's scope are the oxygen vacancy formation, oxygen adsorption and oxygen incorporation kinetics on the (La,Sr) $\mathrm{CoO}_{3}$ films. ${ }^{27,30,33} \mathrm{La}_{2} \mathrm{CoO}_{4+\delta}$ has not been a subject of similar studies to date, and we address the potential effects of strain also in this material as part of this paper.

Another factor that can affect the ORR activity near the $\mathrm{LSC}_{113} / \mathrm{LSC}_{214}$ interface is the redistribution of oxygen defects and dopants. Very recently, Gadre et al., based on DFT calculations, proposed that the enhancement of interfacial ORR activity in this system could be caused by significant $\mathrm{Sr}$ interdiffusion from $\mathrm{LSC}_{113}$ into $\mathrm{LSC}_{214} \cdot{ }^{34}$ The large extent of $\mathrm{Sr}$ enrichment in $\mathrm{LSC}_{214}$ was expected to induce the formation of a large amount of oxygen vacancies and accelerate the oxygen incorporation kinetics. ${ }^{34}$ While this is a reasonable hypothesis, in our ongoing experimental work ${ }^{35}$ we do not find any evidence to a detectable amount of $\mathrm{Sr}$ segregation in $\mathrm{LSC}_{214}$ near the $\mathrm{LSC}_{113} / \mathrm{LSC}_{214}$ interface. Furthermore, it is not clear how the increased amount of oxygen vacancies in $\mathrm{LSC}_{214}$ is to enhance the ORR activity by several orders of magnitude since the interstitial path into $\mathrm{LSC}_{214}$ and similar RP phase compounds is already very fast. Therefore, in this study, we focus on the interstitial oxygen 
incorporation and transport as the dominant ORR path on $\mathrm{LSC}_{214}$ with a Sr doping level $\leq 50 \%$, ${ }^{36 \text {, }}$ ${ }^{37}$ rather than a vacancy dominated oxygen incorporation process.

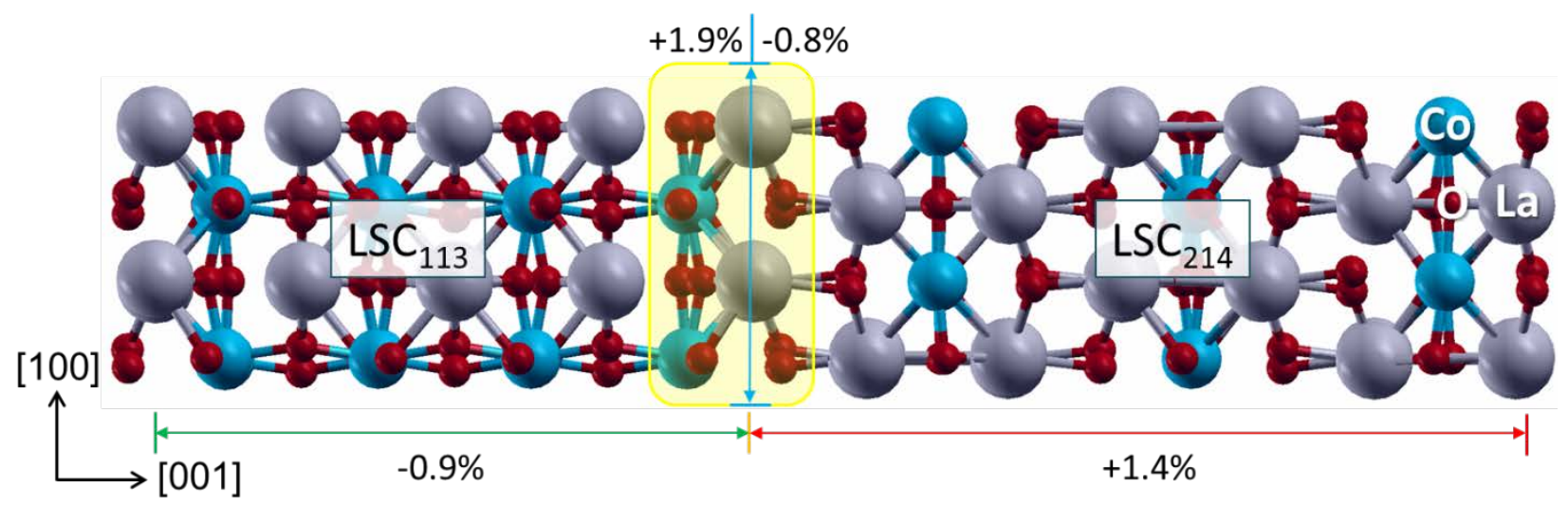

Figure 1. DFT model of the $(\mathrm{La}, \mathrm{Sr}) \mathrm{CoO}_{3}\left(\mathrm{LSC}_{113}\right) /(\mathrm{La}, \mathrm{Sr})_{2} \mathrm{CoO}_{4}\left(\mathrm{LSC}_{214}\right)$ structure, and the theoretically estimated strain states induced near this interface.

In this paper, we quantitatively probe the two above-discussed mechanisms for the reported enhancement of the ORR activity at the hetero-interface of $\mathrm{LSC}_{113} / \mathrm{LSC}_{214}$. In Sec. 2, the computational approach and method details are described. In Sec. 3, first the anisotropic oxygen adsorption, dissociation/incorporation and diffusion on $\mathrm{LSC}_{214}$ as a function of $\mathrm{Sr}$ content, and then the strain effects on the oxygen incorporation and migration kinetics on both $\mathrm{LSC}_{113}$ and $\mathrm{LSC}_{214}$ are assessed. We integrate these two individual effects on each single phase, and estimate the ORR activity enhancement that they together can impose near the LSC $_{113} / \mathrm{LSC}_{214}$ hetero-interface. Finally, in Sec. 4, we summarize our major observations and conclusions.

\section{Computational method and model}

\subsection{Ground-state structures and energies}

We performed plane wave DFT calculations using the Vienna ab initio simulation package (VASP). ${ }^{38}$ We employed the generalized gradient approximation (GGA) parameterized by Perdew and Wang ${ }^{39}$ along with the projector augmented wave (PAW) ${ }^{40}$ method to describe ionic cores. To avoid the self-interaction errors that occur in the traditional DFT for strongly correlated electronic systems, we employed the DFT $+U$ method within Dudarev's approach ${ }^{41}$ 
accounting for the on-site Coulomb interaction in the localized $d$ orbitals, with an effective U-J = $3.3 \mathrm{eV}$ taken from the previous reports for $\mathrm{LaCoO}_{3}{ }^{27,} 30$ and $\mathrm{La}_{2} \mathrm{CoO}_{4+\delta} \cdot{ }^{13}$ For $\mathrm{La}$, Co, and O, the standard PAW/PW91 potentials were used for our calculations, while for Sr, Sr_sv potential was applied. All calculations used a plane wave expansion with a cutoff of $400 \mathrm{eV}$ and included spin polarization. Our test calculation confirmed that the energy cutoff of $400 \mathrm{eV}$ was well converged within $5 \mathrm{meV}$ in total energy, compared to the one with $600 \mathrm{eV}$. Geometries were relaxed using a conjugate gradient algorithm until the forces on all unconstrained atoms were less than 0.03 $\mathrm{eV} / \mathrm{A}$.

The lattice constant of the fully-relaxed bulk $\mathrm{La}_{2} \mathrm{CoO}_{4}$ model is $3.90 \AA(a, b)$ and $12.6 \AA$ (c), which is in good agreement with the experimentally reported values. ${ }^{42}$ To replicate the interfacial strain conditions that the thin films are subjected to at the $\mathrm{LSC}_{113} / \mathrm{LSC}_{214}$ interface, a 2D-planar compressive (tensile) lattice strain was imposed by contracting (elongating) the simulation cell in the [100] and [010] directions and relaxing the cell configuration and dimension in the [001] direction for $\mathrm{LSC}_{214}\left(\mathrm{LSC}_{113}\right)$ in a slab model configuration. The bulk structure of $\mathrm{LSC}_{214}$ was cleaved along the (100) and (001) planes to construct surfaces that are represented by slabs of $\sim 10$ and $11 \AA \AA$ thick, respectively, containing 6 atomic asymmetric layers with the bottom two layers constrained to bulk lattice positions. For all calculations, a vacuum spacing of $\sim 10 \AA$ was placed in the direction of the surface normal. This vacuum spacing, equivalent to the slab thickness, is converged to within $0.5 \mathrm{meV} /$ atom compared to analogous calculations using larger vacuum gaps of 15 and $20 \AA \AA$. When examining adsorption, molecules were placed on only one side of the slab. To avoid the fictitious dipole moment, dipole corrections $^{33,34}$ were applied in computing all of the energies reported here. ${ }^{43,44} \mathrm{O}_{2}$ adsorption calculations were performed for one molecule per surface unit cell, corresponding to a surface coverage of $25 \%$ with four equivalent adsorption sites. A $2 \times 2 \times 1$ Monkhorst-Pack $k$-point mesh was used, which was sufficient to give well converged results. Figure 2 shows the top and side views of the $\operatorname{LSC}_{214}(100)$ unit cell. The details of $\operatorname{LSC}_{113}(001)$ slab model are described in our previous report. ${ }^{27}$ To obtain the $\mathrm{LSC}_{113} / \mathrm{LSC}_{214}$ hetero-structure, we merged the two single-phase slabs in a multilayer configuration (Fig. 1) and fully relaxed both the lattice vectors and atomic positions. For this calculation, we increased the energy cutoff to $600 \mathrm{eV}$ for expanding the wave function and used $\Gamma$ points in the Brillouin zone. 


\subsection{Reaction and migration energy barriers}

The climbing image nudged elastic band (CI-NEB) method $^{45}$ was employed to calculate the migration and incorporation energy barrier of oxygen on and in the $\mathrm{LSC}_{214}$. Initial approximations to reaction paths were obtained by linear interpolation between the energy minima configurations. Three intermediate images were used for all NEB calculations, which was sufficient to map the minimum energy path (MEP) accurately. The NEB simulations were conducted with fixed lattice vectors.

\subsection{Reaction kinetics at the interface}

In order to estimate the relative enhancement in the ORR kinetics near the $\mathrm{LSC}_{113}$ and $\mathrm{LSC}_{214}$ interface compared to the single phase of $\mathrm{LSC}_{113}$, we compute the ratio, $\frac{k_{113 / 214}^{*}}{k_{113}^{*}}$. The surface reaction rate constants, $k^{*}$, are determined by the $\mathrm{O}_{2}$ adsorption and dissociation processes, considered to take place consecutively here, to ultimately incorporate oxygen into the subsurface of each phase.

(a)

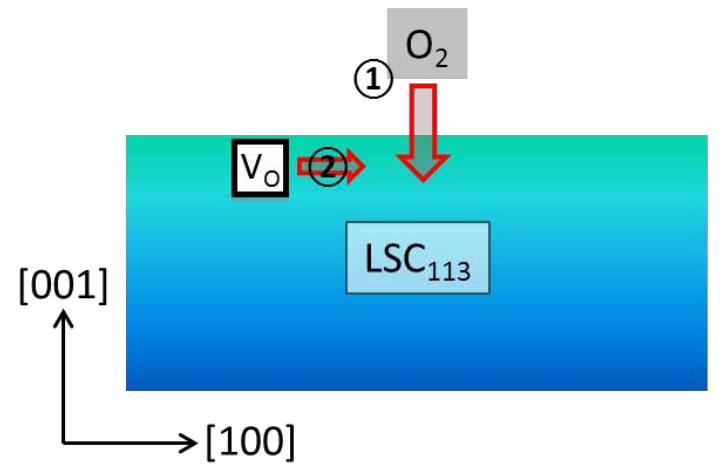

(b)

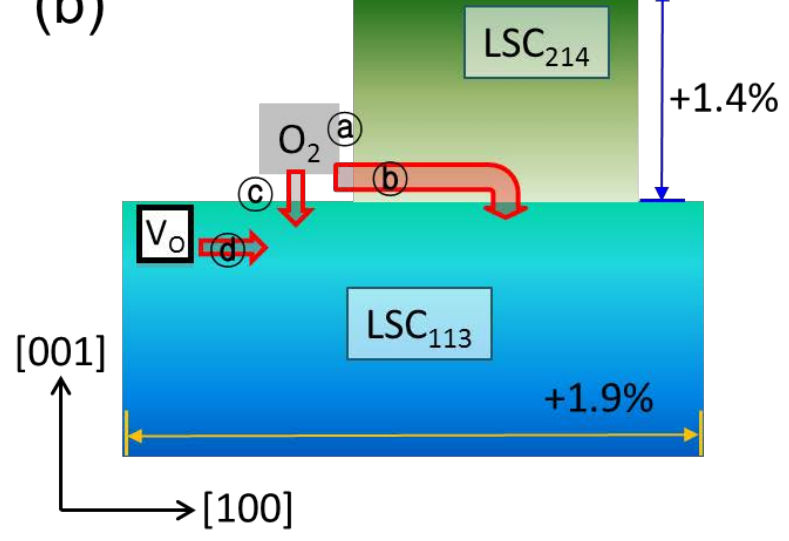

Figure 2. Illustration of the oxygen incorporation paths (a) on the single phase $\mathrm{LSC}_{113}$ and (b) near the $\mathrm{LSC}_{113} / \mathrm{LSC}_{214}$ hetero-interface. The latter includes both path I (a $\rightarrow$ b) and path II (c $\rightarrow$ d).

The oxygen incorporation path on the single phase $\mathrm{LSC}_{113}$ is shown in Fig. 2(a); ${ }^{27} \mathrm{O}_{2}$ adsorbs on the $\mathrm{LSC}_{113}(001)$ surface, and then is incorporated and dissociated by the assistance of a migrating surface oxygen vacancy. For the $\mathrm{LSC}_{113} / \mathrm{LSC}_{214}$ hetero-structure, there are two 
parallel paths for oxygen incorporation as shown in Fig. 2(b); path I (a $\rightarrow$ b) and path II (c $\rightarrow$ d). In path I, oxygen adsorbs on $\mathrm{LSC}_{214}(100)$ surface, then dissociates, incorporates, and migrates through the $\mathrm{LSC}_{214}$ into the $\mathrm{LSC}_{113}$. A path that starts with the $\mathrm{O}_{2}$ adsorption on the $\mathrm{LSC}_{214}(001)$ surface followed by diffusion along the [001] direction is ignored due to very high energy barriers involved, as will be discussed later. The path II is same as the oxygen incorporation path on the single phase $\mathrm{LSC}_{113}$. As described in Fig. 1, presence of lattice strain is expected near the interfaces of this hetero-structure (Fig. 2(b)). In the next sections, we assess the energetics of each unit process along these oxygen incorporation paths, with and without strain, to estimate the enhancement of ORR activity. Here we describe how each process energetics and kinetics are treated in this analysis, especially how we get to the effective $k^{*}$ for each path shown in Fig. 2.

The adsorption energy, $E_{a d s}$, of $\mathrm{O}_{2}$ was defined as

$$
E_{\text {ads }}=E_{\text {total }}-\left(E_{\text {surf }}+E_{\mathrm{O}_{2}}\right)
$$

where $E_{\text {total }}$ is the total energy of the system with the adsorbed $\mathrm{O}_{2}, E_{\text {surf }}$ is the total energy of the bare surface, and $E_{\mathrm{O}_{2}}$ is the total energy for the $\mathrm{O}_{2}$ in the gas phase. With this definition, negative adsorption energies correspond to energetically favored states.

The oxygen vacancy and interstitial formation energies, $E_{v a c}$ and $E_{i n t}$, were calculated as

$$
E_{\text {vac }}=E_{\text {defect }}-E_{0}+\frac{1}{2} E_{\mathrm{O}_{2}}, E_{\text {int }}=E_{\text {defect }}-E_{0}-\frac{1}{2} E_{\mathrm{O}_{2}}
$$

where $E_{\text {defect }}$ is the total energy of the system with a point defect (oxygen vacancy or interstitial) and $E_{0}$ is the total energy of the defect-free system. Smaller formation energy favors the formation of oxygen vacancies or interstitials more easily.

Oxygen incorporation kinetics into the surface of an SOFC cathode are formulated as: ${ }^{46}$

$$
j=-\left.k^{*} \delta c\right|_{\text {surface }}
$$

where $j$ is the flux, $c$ the concentration, $k^{*}$ the effective surface incorporation rate constant of oxygen. The $k^{*}$ is used as a quantitative measure of ORR kinetics here and is approximately calculated as: ${ }^{47}$

$$
k^{*} \approx A_{k} \cdot \exp \left\{-\frac{\left(E_{a d s}+E_{d i s}\right)}{k_{B} T}\right\}
$$


where $A_{k}$ is the prefactor, $E_{a d s}$ the $\mathrm{O}_{2}$ adsorption energy, $E_{d i s}$ the $\mathrm{O}_{2}$ dissociation energy, $k_{B}$ the Boltzmann constant, and $T$ the temperature. For $\mathrm{LSC}_{214}$, the most favorable surface dissociation for $\mathrm{O}_{2}$ involves adsorption in a surface vacancy near the rock-salt layer and dissociation via interstitial incorporation from that site. Therefore, $E_{d i s}$ is treated as the superimposition of oxygen vacancy formation energy $\left(E_{v a c}\right)$ and oxygen incorporation energy barrier $\left(E_{\text {inc }}\right)$ (see Section 3.1). For $\mathrm{LSC}_{113}$, the $\mathrm{O}_{2}$ dissociation process is governed by the availability and mobility of oxygen vacancies on the surface. ${ }^{27,47}$ Therefore, $E_{d i s}$ is composed of the oxygen vacancy formation energy $\left(E_{v a c}\right)$ and oxygen vacancy migration energy $\left(E_{m i g}\right)$ on the surface. ${ }^{27,48}$

\section{Results and Discussion}

\subsection{Anisotropic oxygen adsorption, dissociation and incorporation on $(\mathrm{La}, \mathrm{Sr})_{2} \mathrm{CoO}_{4}$}

We assess the results on both undoped $\mathrm{La}_{2} \mathrm{CoO}_{4}$ (as a model reference system) and on the $50 \%$ Sr-doped $\left(\mathrm{La}_{0.5} \mathrm{Sr}_{0.5}\right)_{2} \mathrm{CoO}_{4}$ (same as the experimentally demonstrated composition ${ }^{15,16}$ ). In each model, $\operatorname{LSC}_{214}(100)$ surface has only one type of termination which includes both the A-site and the B-site cations exposed to the environment. For the $\mathrm{LSC}_{214}(001)$ surface the $\mathrm{CoO}_{2}$ termination layer is considered, and it is equivalent to the $\mathrm{CoO}_{2}$-terminated $\mathrm{LSC}_{113}(001)$ that has been theoretically ${ }^{\dagger}$ found as the most stable surface on perovskite-type cobaltites. ${ }^{49-51}$

\subsection{1. $\mathrm{La}_{2} \mathrm{CoO}_{4}$}

The most stable adsorption structures of $\mathrm{O}_{2}$ on both the (100) and (001) surfaces of undoped $\mathrm{LSC}_{214}$ are shown in Fig. 3(a) and (b), respectively. An $\mathrm{O}_{2}$ molecule adsorbs onto the bridge site of $\operatorname{LSC}_{214}(100)$ between La atoms in the vicinity of the interstitial path between the LaO planes. Surface oxygen vacancies are not expected to be present on the undoped $\mathrm{LSC}_{214}{ }^{36}$, 37,52 and do not play a role on the $\mathrm{O}_{2}$ adsorption and dissociation on the (100) surface. Interestingly, the adsorption energy in this configuration is $-3.24 \mathrm{eV}$, indicating a very strong

\footnotetext{
${ }^{\dagger}$ Recent experimental observations report the presence of the AO-plane terminated surface on LSM and LSC, and this is different from the theoretical (DFT-based) prediction of a stable $\mathrm{BO}_{2}$-surface. We think that, "the relative" changes in surface reaction energies with anisotropy and with lattice strain should not be influenced significantly by this choice of the surface termination. This is because, even if the surface were AO-terminated, the oxygen vacancies are then necessary for the adsorption and dissociation of oxygen, and they reside on top of the Co cations exposed through the vacancies of the top AO-plane. Therefore, even if the $\mathrm{CoO}_{2}$ layer were beneath the surface, it is still likely to play an important role in the chemisorption and dissociation of oxygen molecules on the surface.
} 
adsorption of $\mathrm{O}_{2}$ on this surface. On the other hand, the $\mathrm{O}_{2}$ adsorption energy on $\mathrm{LSC}_{214}(001)$ is $0.24 \mathrm{eV}$, which is considerably weaker. As expected, this value and the adsorption configuration (with the $\mathrm{O}_{2}$ atop a Co atom in a tilted fashion ${ }^{27,30}$ ) are very close to the one on $\mathrm{LSC}_{113}(001){ }^{27}$ The adsorption energy difference between (100) and (001) surfaces on undoped LSC 214 is $3 \mathrm{eV}$, favoring that on the former.

$\mathrm{O}_{2}$ adsorption on $\mathrm{LSC}_{214}(100)$ is followed by rotation and dissociation of $\mathrm{O}_{2}$ that directly leads to $\mathrm{O}$ incorporation as an interstitial into the rock-salt layer, as shown in Fig. 3(c) and the insets of Fig. 3(d). The dissociated $\mathrm{O}$ replaces the lattice oxygen of the surface by kicking it off into an interstitial site between the $\mathrm{LaO}$ planes in the subsurface region (Fig. 3(c)). This is analogous to the interstitialcy path that governs the oxygen migration in the bulk of this material. ${ }^{13}$ The energy barrier for this incorporation process on the undoped $\operatorname{LSC}_{214}(100)$ is 0.77 eV (Fig. 3(d)), which is lower than the one on $\operatorname{LSC}_{113}(001)$ surface $(1.31 \mathrm{eV})$ that is governed by the availability and mobility of surface oxygen vacancy. ${ }^{27}$ Therefore, the $\mathrm{O}_{2}$ dissociation kinetics alone on the $\mathrm{LSC}_{214}$ surface is expected to present an additional enhancement by $0.54 \mathrm{eV}$ compared to that on the $\mathrm{LSC}_{113}$ surface.

Similarly to the other $\mathrm{A}_{2} \mathrm{BO}_{4}$ materials, ${ }^{6,12,13,17-23}$ we quantitatively show here that oxygen diffuses anisotropically in $\mathrm{LSC}_{214}{ }^{13}$ (Fig. 3(e)). The oxygen migration barrier along the [100] direction (by interstitialcy mechanism) in the bulk $\mathrm{LSC}_{214}$ is $0.61 \mathrm{eV}$, which is significantly lower than the $1.95 \mathrm{eV}$ in the [001] direction. The oxygen transport on the $\mathrm{LSC}_{214}(100)$ surface in the [001] direction is even more unfavorable with an energy barrier of $\sim 3 \mathrm{eV}$ (Fig. 3(f)). In $\mathrm{LSC}_{214}$, therefore, oxygen most favorably diffuses along the [100] direction via the interstitialcy path. ${ }^{13}$ The kinetics of oxygen diffusion in $\mathrm{LSC}_{214}$, however, does not significantly accelerate the overall ORR kinetics compared to $\mathrm{LSC}_{113}$ because the migration barrier of oxygen in bulk $\operatorname{LSC}_{113}(0.69 \mathrm{eV})^{27}$ is similar to the one along the fast interstitialcy route in LSC 214 . Since the focus is on "surface incorporation rate of oxygen" on $\operatorname{LSC}_{214}(100)$, oxygen diffusion in the bulk along the [001] direction was not included into the quantitative estimate of the relative enhancement of ORR kinetics based on $k^{*}$. Furthermore, at the hetero-interface of $\mathrm{LSC}_{113} / \mathrm{LSC}_{214}$, the fast diffusion path in [100] direction is in direct contact with $\mathrm{LSC}_{113}$, thus oxygen can directly incorporate into $\mathrm{LSC}_{113}$ without the diffusion step across the rock-salt layers along the [001] in LSC 214 . Lastly, even if a small number of migration steps along the [001] direction near the interface takes place, the overall "resistance" of this very short path would be small despite 
the relatively large energy barrier. These points justify that diffusion along [001] direction is not a significant contributor to the ORR assessment.
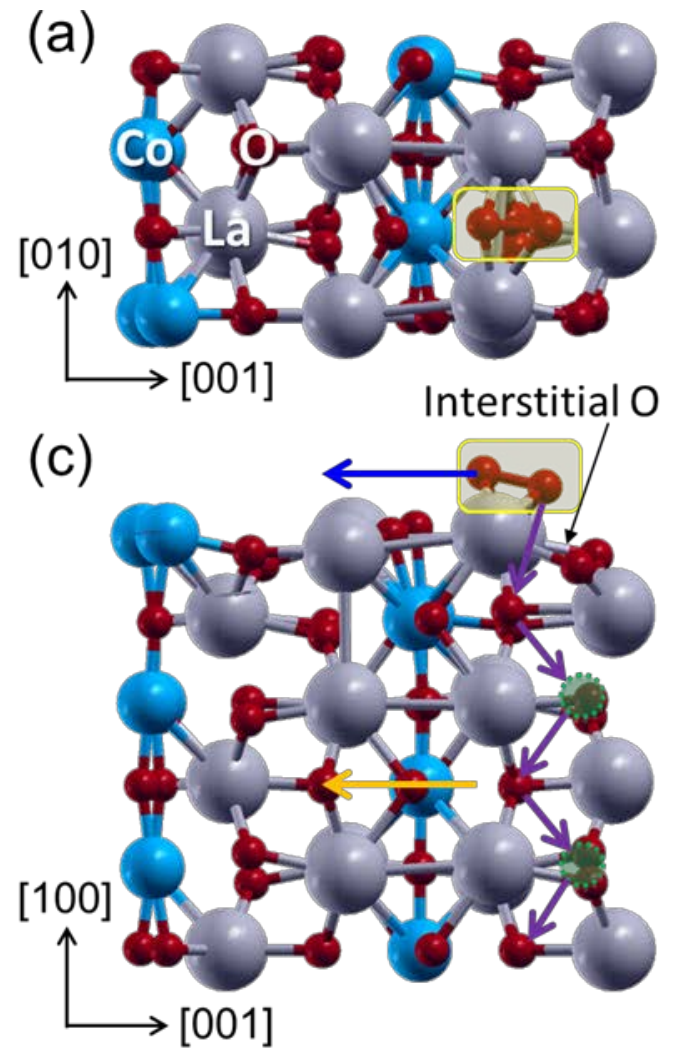

(e)

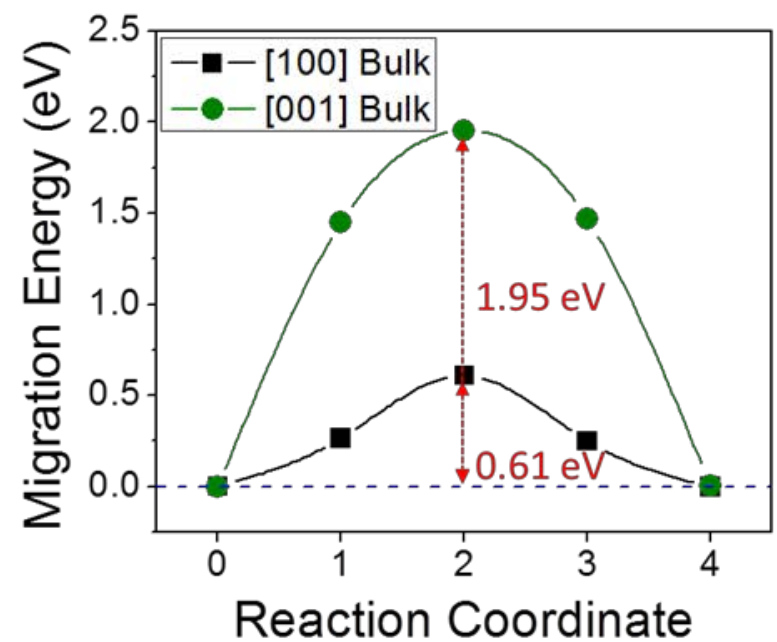

(b)

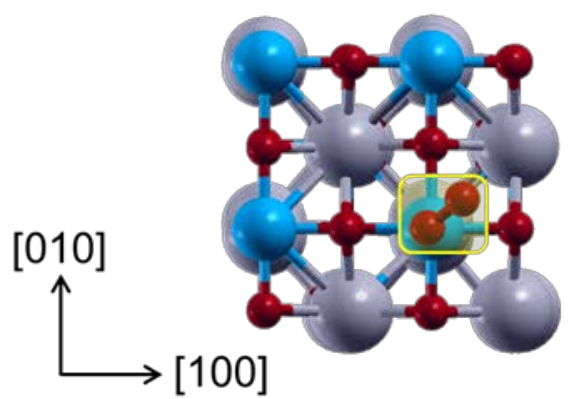

(d)
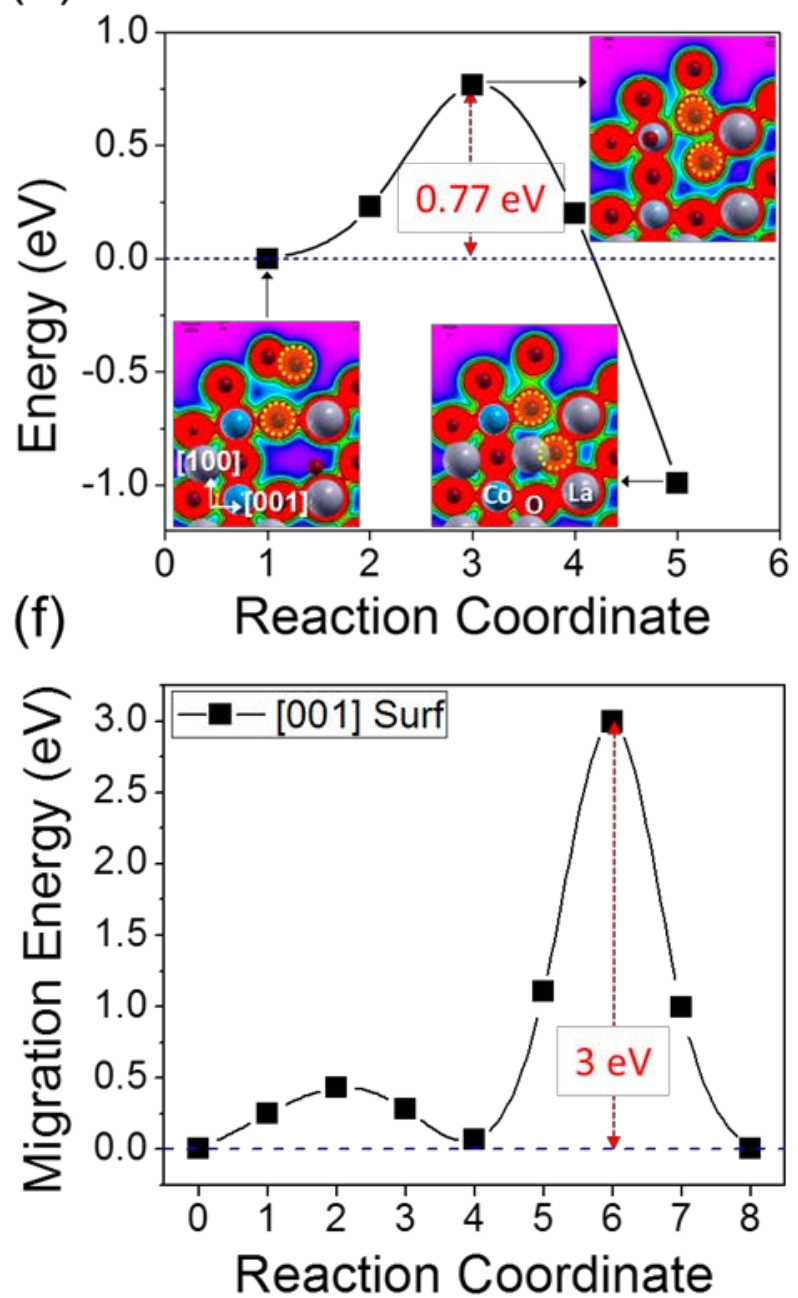

Figure 3. Top view of (a) $\mathrm{La}_{2} \mathrm{CoO}_{4}(100)$, (b) $\mathrm{La}_{2} \mathrm{CoO}_{4}(001)$, (c) side view of $\mathrm{La}_{2} \mathrm{CoO}_{4}$ model unit cell which schematically shows the oxygen migration pathways along the [001] on the 
surface (blue arrow) and in the bulk (yellow arrow), and along the [100] direction into the bulk (purple arrow), and (d) the relative energy of the system for $\mathrm{O}_{2}$ dissociation/incorporation on $\mathrm{La}_{2} \mathrm{CoO}_{4}$ along the [100] direction. The yellow boxes in (a-c) show the adsorbed oxygen molecule on each surface. Charge density plots for the initial, transition, and final states are shown in the insets of (d) for oxygen incorporation. The relative energy of the system for (e) $\mathrm{O}$ migration along the [100] direction (purple arrows in (c)) and along the [001] direction (orange arrow in (c)) in the bulk $\mathrm{La}_{2} \mathrm{CoO}_{4}$; and for (f) $\mathrm{O}$ migration on $\mathrm{La}_{2} \mathrm{CoO}_{4}(100)$ surface (blue arrow in (c)). The $3 \mathrm{eV}$ barrier in (f) corresponds to the migration of oxygen across the AO-AO rocksalt layers on the $\mathrm{La}_{2} \mathrm{CoO}_{4}(100)$ surface (Fig. S1 in the Supplementary Information).

\subsection{2. $\left(\mathrm{La}_{0.5} \mathrm{Sr}_{0.5}\right)_{2} \mathrm{CoO}_{4}$}

For assessing the oxygen incorporation kinetics on the $50 \%$ Sr-doped $\mathrm{LSC}_{214}$, one must know the most stable Sr distribution in the structure. We compared the energetic stability of LSC $_{214}$ with a uniform distribution of Sr and with a cation ordered structure. In the latter, double layers of $\mathrm{SrO}$ and $\mathrm{LaO}$ are 1:1 ordered perpendicular to the $c$ axis as shown in Fig. 4(a) and (c). This layered structure of the A-site cations has also been observed in similar RP phase systems. ${ }^{53-60}$ Our DFT calculations showed that this cation ordered LSC $_{214}$ is energetically more stable than the one with uniform distribution of Sr, by $0.04 \mathrm{eV} /$ formula unit (f.u.) (See Fig. S2 in the Supplementary Information). We therefore evaluated the oxygen adsorption, dissociation, and incorporation processes on the cation ordered LSC $_{214}$ model. Multiple pathways for these processes were considered and quantitatively investigated (see Fig. S4 in the Supplementary Information).

Although oxygen incorporation into $\left(\mathrm{La}_{0.5} \mathrm{Sr}_{0.5}\right)_{2} \mathrm{CoO}_{4}$ is not mediated by exchange with surface and subsurface vacancies (as noted in Sec. 1), it is reasonable to expect presence of oxygen vacancies on the (100) surface with as high of a Sr content. ${ }^{36,37,52}$ Vacancy and interstitial formation energies calculated here on various positions on the surface and in the bulk $\mathrm{LSC}_{214}$ justify this (see Fig. S3 in the Supplementary Information). In the bulk, oxygen interstitials are most dominantly favored, with a formation energy of $E_{\text {int }}=0.22 \mathrm{eV}$, over oxygen vacancies with $E_{v a c}=2 \mathrm{eV}$. Thus, oxygen incorporation into and diffusion in the bulk $\left(\mathrm{La}_{0.5} \mathrm{Sr}_{0.5}\right)_{2} \mathrm{CoO}_{4}$ take place via oxygen interstitials, similarly to the case of undoped $\mathrm{LSC}_{214}$. On the $\left(\mathrm{La}_{0.5} \mathrm{Sr}_{0.5}\right)_{2} \mathrm{CoO}_{4}(100)$ surface, however, the surface oxygen interstitial is not stable. Oxygen 
rather preferentially binds on the bridge site between La atoms on the rock-salt layer; this is 0.52 $\mathrm{eV}$ more favorable than the binding onto the interstitial site on the (100) surface. Vacancy formation energy on this surface varies as $2.15 \sim 3.60 \mathrm{eV}$ depending on the vacancy site considered. Without surface oxygen interstitials, it is possible that the surface vacancies (with formation energy of $2.15 \mathrm{eV}$ ) can exist on the AO-AO rock-salt layers at elevated temperatures. Although their concentration is not expected to be very high, their presence is considered crucial upon surveying the oxygen reaction pathways on the surface.

Among the oxygen incorporation pathways considered on $\left(\mathrm{La}_{0.5} \mathrm{Sr}_{0.5}\right)_{2} \mathrm{CoO}_{4}(100)$, the one in which an $\mathrm{O}_{2}$ first adsorbs into a surface vacancy on the rock-salt layer (position 2 in Fig. S3(c) in the Supplementary Information), followed by oxygen dissociation and incorporation into an interstitial site in the rock-salt layer is energetically the most favorable (Fig. 4(c)). This is unlike the case on the undoped $\mathrm{LSC}_{214}(100)$ where the most preferred $\mathrm{O}_{2}$ adsorption site is the bridging site between La atoms on the rock-salt layer. Although the adsorption on $\left(\mathrm{La}_{0.5} \mathrm{Sr}_{0.5}\right)_{2} \mathrm{CoO}_{4}(100)$ is into a vacancy, the dissociation is into an interstitial site between the rock-salt layers, as shown in the insets of Fig. 4(d), and would be followed by interstitialcy diffusion. $2.15 \mathrm{eV}$ is required for vacancy formation and $0.83 \mathrm{eV}$ for oxygen dissociation and incorporation barrier. The energy barrier for this incorporation process on 50\% Sr-doped $\operatorname{LSC}_{214}(100)$ is slightly higher than that on undoped $\mathrm{LSC}_{214}(100)(0.77 \mathrm{eV})$ and on $\mathrm{LSC}_{113}(001)$ surface $(0.70 \mathrm{eV}) .{ }^{27}$ This suggests that the increase of Sr content on $\mathrm{LSC}_{214}$ does not further facilitate the oxygen incorporation.

Upon doping Sr into La sites, the adsorption strength on the $\operatorname{LSC}_{214}(100)$ surface reduces relatively, while the adsorption energy and configuration on the $\operatorname{LSC}_{214}(001)$ surface remains almost the same (due to lack of A-site cations on that surface). Despite the adsorption into an oxygen vacancy on the $50 \%$ Sr-doped $\operatorname{LSC}_{214}(100)$, the adsorption energy is $-2.02 \mathrm{eV}$, and this is $1.22 \mathrm{eV}$ weaker than the case on the undoped one. This decreases the adsorption energy difference between the (100) and (001) surfaces from $3 \mathrm{eV}$ for undoped $\mathrm{LSC}_{214}$ to $1.82 \mathrm{eV}$ for $50 \%$ Sr-doped $\mathrm{LSC}_{214}$, respectively (Table 1), but the $1.82 \mathrm{eV}$ that represents the anisotropy of oxygen adsorption is still very significant. The relative reduction in the $\mathrm{O}_{2}$ adsorption strength upon $\mathrm{Sr}$ doping can be explained as follows. On an undoped $\operatorname{LSC}_{214}(100)$, significant surface relaxations take place to accommodate the adsorbing oxygen. On the 50\% Sr-doped surface, however, the relatively large Sr atoms inhibit the relaxation of the top layer of the (100) surface and retain a 
rigid structure (Fig. 4(a)) upon $\mathrm{O}_{2}$ adsorption. Overall such strongly enhanced adsorption on the (100) surface of $\mathrm{LSC}_{214}$ can provide a large flux of oxygen as an increased source term for the oxygen incorporation kinetics on $\operatorname{LSC}_{214}(100)$, and is one possible reason for the ORR enhancement at the hetero-interface of $\mathrm{LSC}_{113} / \mathrm{LSC}_{214} \cdot{ }^{15,16}$

Our results suggest that doping of $\mathrm{Sr}$ into $\mathrm{LSC}_{214}$ hinders the oxygen adsorption and incorporation process relative to the undoped $\mathrm{LSC}_{214}$ due to structural reasons. However, electron transfer properties of Sr-doped $\mathrm{LSC}_{214}$ at finite temperatures is actually expected to help the overall reaction kinetics. Furthermore, for both $0 \%$ and $50 \%$ Sr-doped $\mathrm{LSC}_{214}$, the anisotropically strong adsorption of $\mathrm{O}_{2}$ on $\mathrm{LSC}_{214}(100)$ plays a more important role than the anisotropic oxygen dissociation and incorporation into $\mathrm{LSC}_{214}$ for the ORR enhancement near the $\mathrm{LSC}_{113} / \mathrm{LSC}_{214}$ hetero-interface.

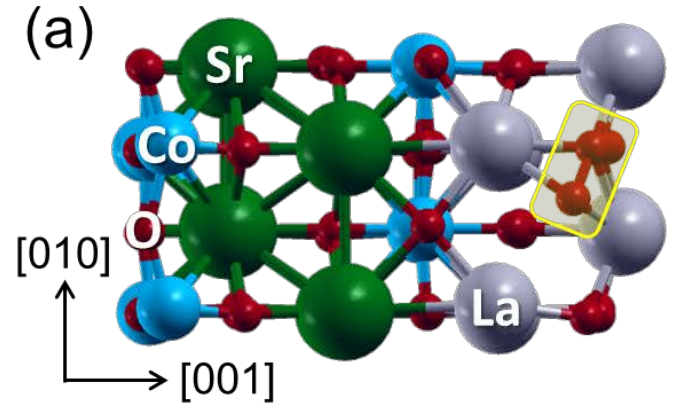

(c)

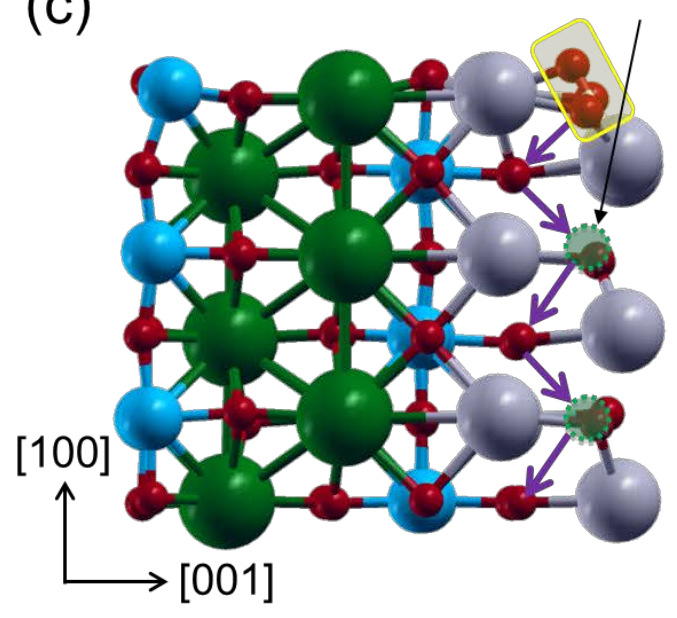

(b)

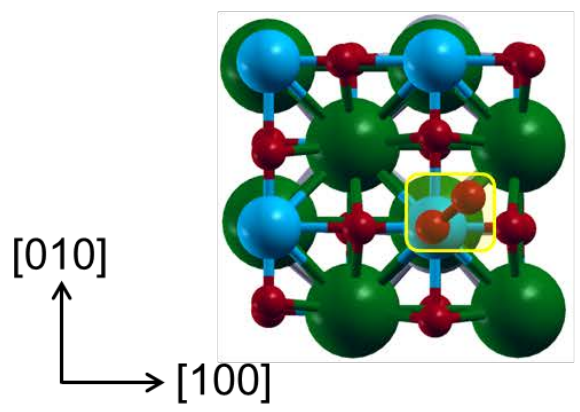

(d)

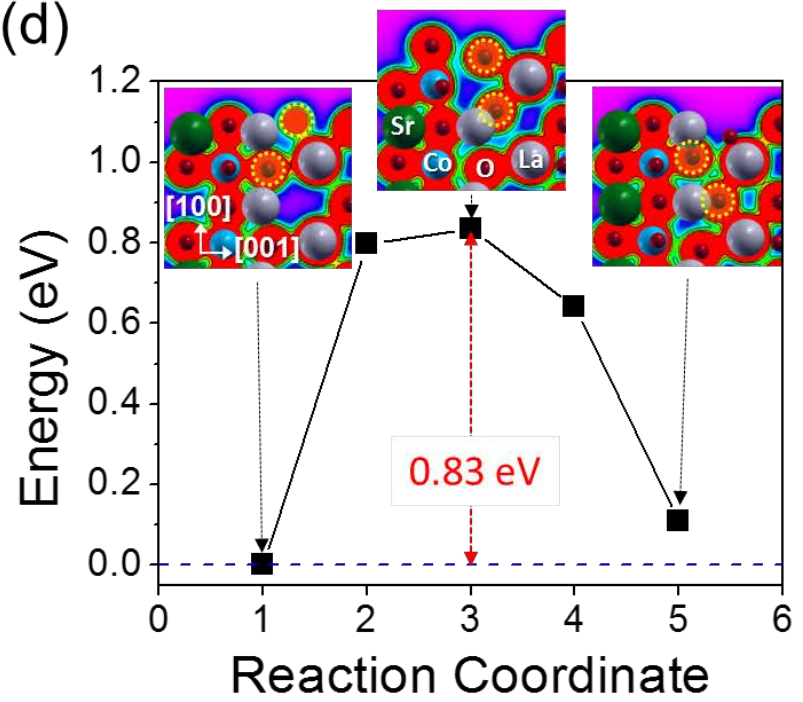

Figure 4. Top view of (a) $\left(\mathrm{La}_{0.5} \mathrm{Sr}_{0.5}\right)_{2} \mathrm{CoO}_{4}(100)$, (b) $\left(\mathrm{La}_{0.5} \mathrm{Sr}_{0.5}\right)_{2} \mathrm{CoO}_{4}(001)$, (c) side view of $\left(\mathrm{La}_{0.5} \mathrm{Sr}_{0.5}\right)_{2} \mathrm{CoO}_{4}$ model unit cell which schematically shows the oxygen incorporation and 
migration pathway along the [100] direction into the bulk (purple arrow), and (d) the relative energy of the system for oxygen incorporation into $\left(\mathrm{La}_{0.5} \mathrm{Sr}_{0.5}\right)_{2} \mathrm{CoO}_{4}$ along the [100] direction. The yellow boxes in (a), (b) and (c) show the adsorbed oxygen molecule on each surface. Adsorption is into an oxygen vacancy on the AO-AO rock-salt layer in (a) and (c). Charge density plots for the initial, transition, and final states for oxygen incorporation are shown in the insets of (d).

\begin{tabular}{|c||c|c|}
\hline $\boldsymbol{E}_{\text {ads }} \mathbf{( e V )}$ & $\mathbf{L a}_{\mathbf{2}} \mathrm{CoO}_{\mathbf{4}}$ & $\left.\mathbf{( L a}_{\mathbf{0 . 5}} \mathrm{Sr}_{\mathbf{0 . 5}}\right)_{\mathbf{2}} \mathbf{C o O}_{\mathbf{4}}$ \\
\hline \hline $\mathbf{( 1 0 0 )}$ & -3.24 & -2.02 \\
\hline $\mathbf{( 0 0 1 )}$ & -0.24 & -0.20 \\
\hline Difference & 3.00 & 1.82 \\
\hline
\end{tabular}

Table 1. Adsorption energies of oxygen molecule on the (100) and (001) surfaces of $0 \%$ and $50 \%$ Sr-doped LSC 214 . The 'Difference' represents the anisotropic adsorption strength that favors the (100) surface.

\subsection{Strain effect on the oxygen incorporation on $(\mathrm{La}, \mathrm{Sr}) \mathrm{CoO}_{3}$ and $(\mathrm{La}, \mathrm{Sr})_{2} \mathrm{CoO}_{4}$ near the hetero-interface}

\subsection{1. ( $\mathrm{La}, \mathrm{Sr}) \mathrm{CoO}_{3}$}

As described in Figs. 1 and 2, $\operatorname{LSC}_{113}(001)$ is in theory up to $+1.9 \%$ tensile strained in the [100] and [010] directions near the $\mathrm{LSC}_{113} / \mathrm{LSC}_{214}$ interface. Thus, we can include here the effect of strain on oxygen adsorption, and vacancy formation and migration on the $\mathrm{LSC}_{113}(001)$ surface, which we previously assessed. ${ }^{27,30}$ We remind that the oxygen dissociation and incorporation kinetics is limited by the availability and mobility of oxygen vacancies on the surface of $\mathrm{LSC}_{113}$, as well as on other similar perovskite cathodes. ${ }^{27,48}$ As the tensile strain increases up to the limit of elastic stretching, both the oxygen molecule adsorption and oxygen vacancy formation become more favored, as shown in Fig. 5(a). ${ }^{27}$ Increasing planar tensile strain weakens the in-plane Co-O bonds by decreasing the Co $d$ and lattice $\mathrm{O} p$ orbitals' hybridization, which consequently causes to strengthen the chemisorption of $\mathrm{O}_{2}$ onto Co. Oxygen vacancy is more easily formed due to the weakening of the in-plane Co-O bonds upon tensile strain. The energy barrier for oxygen vacancy migration on $\operatorname{LSC}_{113}(001)$ is significantly lowered in the [1 $\overline{1} 0$ ] direction with the increase of strain, as shown in Fig. 5(b), ${ }^{27}$ because of the increased space 
available in the migration path of oxygen. We will use this strain dependence later to quantify the acceleration of the ORR kinetics near the hetero-interface of $\mathrm{LSC}_{113} / \mathrm{LSC}_{214}$ in Sec. 3.3.

We note here that, because the $\mathrm{LSC}_{113}$ (001) surface investigated here is $\mathrm{CoO}_{2}$-terminated, the Sr-dopant level does not introduce significant quantitative differences to our results, especially with regards to the relative effects of strain. Thus, all the LSC113 results reported and used in this paper are taken on undoped LSC113.
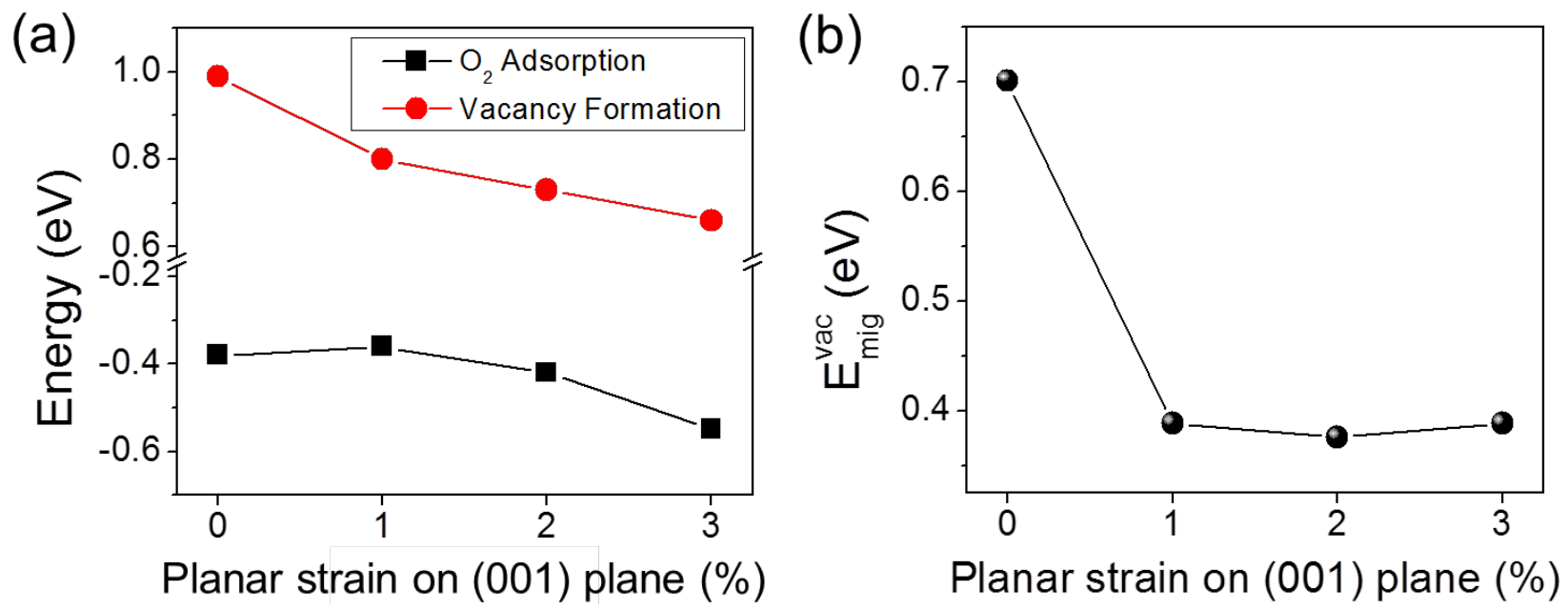

Figure 5. (a) Tensile planar strain dependence of (a) $\mathrm{O}_{2}$ adsorption and oxygen vacancy formation energies and of (b) migration energy barrier of oxygen vacancy in the [1 10 ] direction on undoped $\operatorname{LSC}_{113}(001)$ surface. (reprinted with permission from Ref. 27)

\subsection{2. $\left(\mathrm{La}_{0.5} \mathrm{Sr}_{0.5}\right)_{2} \mathrm{CoO}_{4}$}

Theoretically estimated strain in $\mathrm{LSC}_{214}$ near the $\mathrm{LSC}_{113} / \mathrm{LSC}_{214}$ hetero-interface is $-0.8 \%$ compressive planar strain on (001) plane, which leads to +1.4 tensile strain relaxation in [001] direction (Fig.(1)). Experimentally, in a multilayer $\mathrm{LSC}_{113} / \mathrm{LSC}_{214}$ heterostructure the largest strain that is measured in $\mathrm{LSC}_{214}$ layers is $+1 \%$ tensile strain along $[001],{ }^{35}$ which arises from a $1.5 \%$ compressive planar strain on (001) plane. The latter case is considered here to represent the maximum feasible strain in such a structure for the evaluation of strain effects on LSC $_{214}$. As shown in Fig. 6(a), the strain dependence of $\mathrm{O}_{2}$ adsorption and vacancy formation is very weak, with a change of only $0.06 \mathrm{eV}$ and $0.05 \mathrm{eV}$, respectively, at $-1.5 \%$ compared to the unstrained state. The strain response of oxygen incorporation into $\mathrm{LSC}_{214}$ along the [100] direction (Fig. 
4(c)) is more significant, with a reduction of incorporation energy barrier up to $0.21 \mathrm{eV}$. The strain dependence of the oxygen incorporation energy is correlated to the increase in the width of the entrance channel for oxygen interstitial (measured as the distance across the rock-salt layer), Fig. S5 in the supplementary information). Overall, the strain dependence on the single phase $\mathrm{LSC}_{214}$ surface is weaker than the one on the single phase $\mathrm{LSC}_{113}$ surface (Fig. 5).
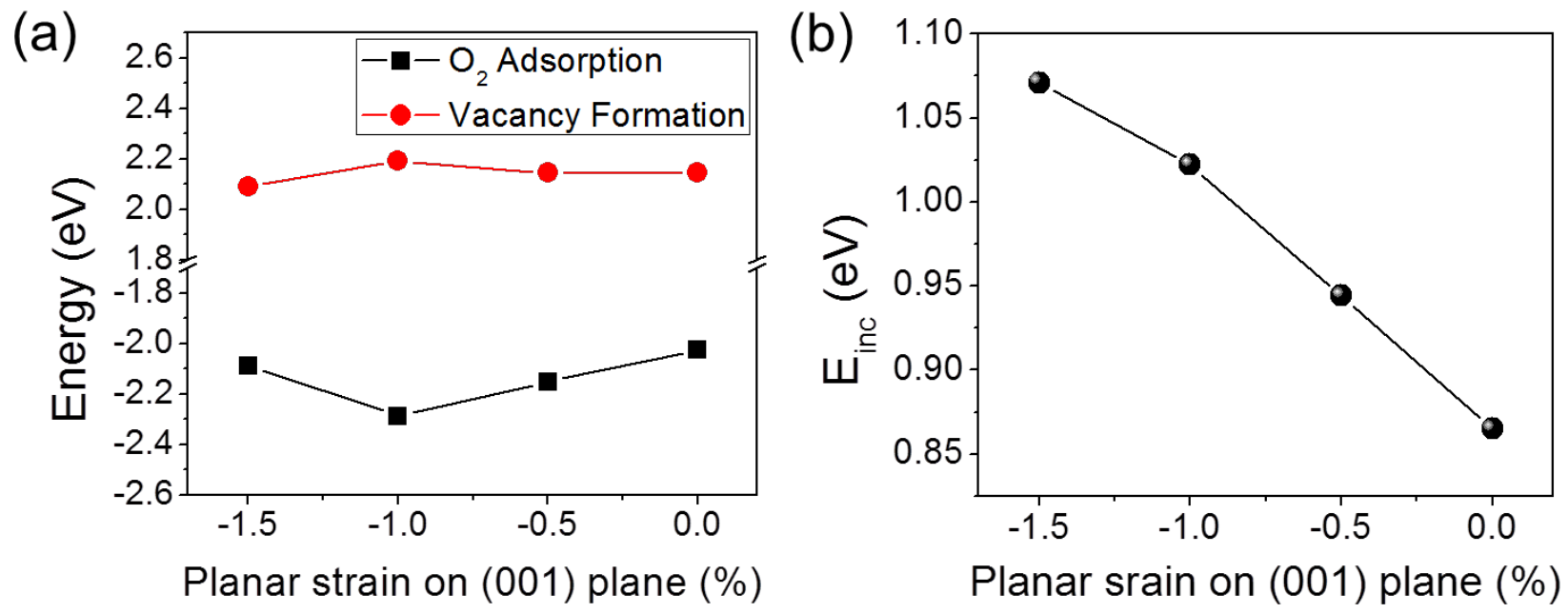

Figure 6. Strain dependence of (a) $\mathrm{O}_{2}$ adsorption and oxygen vacancy formation energies, and (b) the energy barrier of oxygen incorporation into $50 \%$ Sr-doped $\mathrm{LSC}_{214}(100)$ surface.

\subsection{Quantification of the enhancement of oxygen reduction kinetics near the $\mathrm{LSC}_{113} / \mathrm{LSC}_{214}$ hetero-interface}

Here we integrate the individual effects of anisotropy and lattice strain (for each single phase), described in 3.1-3.2 above, to quantitatively estimate the relative enhancement in the ORR kinetics expected near the $\mathrm{LSC}_{113} / \mathrm{LSC}_{214}$ hetero-interface. In Sec. 1, we showed the theoretical lattice strain near the hetero-interface (Fig. 1). The actual strain induced in the $\mathrm{LSC}_{113}$ and LSC $_{214}$ layers in a multilayer configuration in our ongoing experimental work was found by X-ray diffraction. ${ }^{35}$ The experimentally determined strain states along the [100] and [001] directions are consistent qualitatively (in sign) with those predicted by the DFT calculations in this paper. In estimating the maximum effect of lattice strain on the relative ORR kinetics enhancement, we use the largest strain values that are experimentally found: $-1.5 \%$ compressive planar strain on the (001) plane of $\mathrm{LSC}_{214}$ and $+0.8 \%$ tensile planar strain on the (001) plane of 
$\mathrm{LSC}_{113}$. These planar strain values are deduced from the measured $+1.0 \%$ tensile strain in $\mathrm{LSC}_{214}$ and $-0.6 \%$ compressive strain in $\mathrm{LSC}_{113}$ in the [001] direction, using 0.25 as the Poisson ratio typical for most oxides.

We compare the overall oxygen reduction kinetics at/near the $\mathrm{LSC}_{113} / \mathrm{LSC}_{214}$ heterointerface (Fig. 2(b)) with that on the single phase LSC $_{113}$ (Fig. 2(a)) on the basis of the strainand anisotropy-dependent energetics of unit processes discussed in Sec. 3.2. The paths for oxygen incorporation near the hetero-interface were described in Fig. 2 and Sec. 2.3. Table 2 summarizes all energies used for this quantitative comparison reported next.

\begin{tabular}{|c|c|c|c|c|}
\hline Unit process energies $(\mathrm{eV})$ & $E_{a d s}$ & $E_{v a c}$ & $E_{\text {inc }}$ & $E_{\text {mig }}$ \\
\hline Unstrained LSC $_{214}(100)$ & -2.02 & 2.15 & 0.87 & -- \\
\hline Unstrained $\mathrm{LSC}_{113}(001)$ & -0.38 & 0.99 & -- & 0.70 \\
\hline$-1.5 \%$ strained LSC $_{214}$ on (001) plane & -2.09 & 2.09 & 1.07 & -- \\
\hline$+0.8 \%$ strained $\mathrm{LSC}_{113}$ on (001) plane & -0.36 & 0.83 & -- & 0.44 \\
\hline
\end{tabular}

Table 2. Unit reaction energies on the unstrained and strained $\mathrm{LSC}_{113}$ and $\mathrm{LSC}_{214}$. The strain range near the $\mathrm{LSC}_{113} / \mathrm{LSC}_{214}$ hetero-interface is based on our measurements on a multilayer configuration made of $\mathrm{LSC}_{113}$ and $\mathrm{LSC}_{214 \cdot}{ }^{35}$

As explained in Sec. 2, in order to estimate the relative enhancement of oxygen reduction kinetics, we calculate the ratio of the $k^{*}$ on the $\mathrm{LSC}_{113} / \mathrm{LSC}_{214}$ hetero-structure to that on the single phase $\mathrm{LSC}_{113}$. Using Equation (4), the $k^{*}$ via the oxygen incorporation path I (Fig. 2(b)) is:

$$
k_{113 / 214-I}^{*} \approx\left[v_{1} \cdot \exp \left\{-\frac{\left(E_{a d s}^{214}+E_{v a c}^{214}+E_{i n c}^{214}\right)}{k_{B} T}\right\}\right]_{113 / 214} \text {. }
$$

Using Equation (4), the $k^{*}$ via the path II (Fig. 2(b)) is:

$$
k_{113 / 214-I I}^{*} \approx\left[v_{2} \cdot \exp \left\{-\frac{\left(E_{a d s}^{113}+E_{v a c}^{113}+E_{m i g}^{113}\right)}{k_{B} T}\right\}\right]_{113 / 214} \text {. }
$$

All pre-factors in Equations (5) and (6) are incorporated into $v_{1}$ and $v_{2}$, respectively. The $k_{113 / 214}^{*}$ for the $\mathrm{LSC}_{113} / \mathrm{LSC}_{214}$ hetero-structure is the sum of $k_{I}^{*}$ and $k_{I I}^{*}$. 
For the single phase unstrained reference state of $\mathrm{LSC}_{113}, k_{113}^{*}$ is:

$$
k_{113}^{*} \approx\left[v_{3} \cdot \exp \left\{-\frac{\left(E_{a d s}^{113}+E_{v a c}^{113}+E_{m i g}^{113}\right)}{k_{B} T}\right\}\right]_{113},
$$

where all prefactors are incorporated into $v_{3}$. We assume that the prefactor terms, $v_{1}, v_{2}$, and $v_{3}$ are approximately the same on the single phase $\mathrm{LSC}_{113}$ and the $\mathrm{LSC}_{113} / \mathrm{LSC}_{214}$ hetero-structure.

The ratio $\frac{k_{113 / 214}^{*}}{k_{113}^{*}}$ that represents the relative enhancement of ORR kinetics at/near the $\mathrm{LSC}_{113} / \mathrm{LSC}_{214}$ interface compared to that on the single phase $\mathrm{LSC}_{113}$ is calculated using Equations (5)-(7) and Table 2. At $500{ }^{\circ} \mathrm{C}$ representative of Sase et al.'s ${ }^{15}{ }^{18} \mathrm{O}-\mathrm{SIMS}$ measurement conditions $\frac{k_{113 / 214}^{*}}{k_{113}^{*}}$ is found as $4 \times 10^{2}$.

We deconvolute the effect of anisotropy and the effect of strain in this total enhancement factor by subtracting the factor with no strain from the total $\frac{k_{113 / 214}^{*}}{k_{113}^{*}}$ noted above as:

$$
\left[\frac{k_{113 / 214}^{*}}{k_{113}^{*}}\right]_{\text {strain }}=\frac{k_{113 / 214}^{*}}{k_{113}^{*}}-\left[\frac{k_{113 / 214}^{*}}{k_{113}^{*}}\right]_{\text {no strain }} .
$$

We note that the $\left[\frac{k_{113 / 214}^{*}}{k_{113}^{*}}\right]_{\text {no strain }}$ is equivalent to the $\mathrm{LSC}_{214}$ anisotropy effect alone at this hetero-structure. Fig. 7 shows these results in the $400-800{ }^{\circ} \mathrm{C}$ range, and as expected the relative acceleration in ORR is more significant at the lower temperatures. At $500{ }^{\circ} \mathrm{C}$, the contribution to the overall enhancement factor from the $\mathrm{LSC}_{214}$ anisotropy is $10^{2}$ times. The contribution from lattice strain (considered both in $\mathrm{LSC}_{113}$ and $\mathrm{LSC}_{214}$ together) at $500{ }^{\circ} \mathrm{C}$ is $3 \times 10^{2}$ times. On the basis of these results, we think that both the $\mathrm{LSC}_{214}$ anisotropy and the strain effect especially in $\mathrm{LSC}_{113}$ could have acted equally importantly as two possible sources to accelerate the ORR kinetics on the $\mathrm{LSC}_{113} / \mathrm{LSC}_{214}$ hetero-structure that was reported experimentally in refs. 15 and 16. 


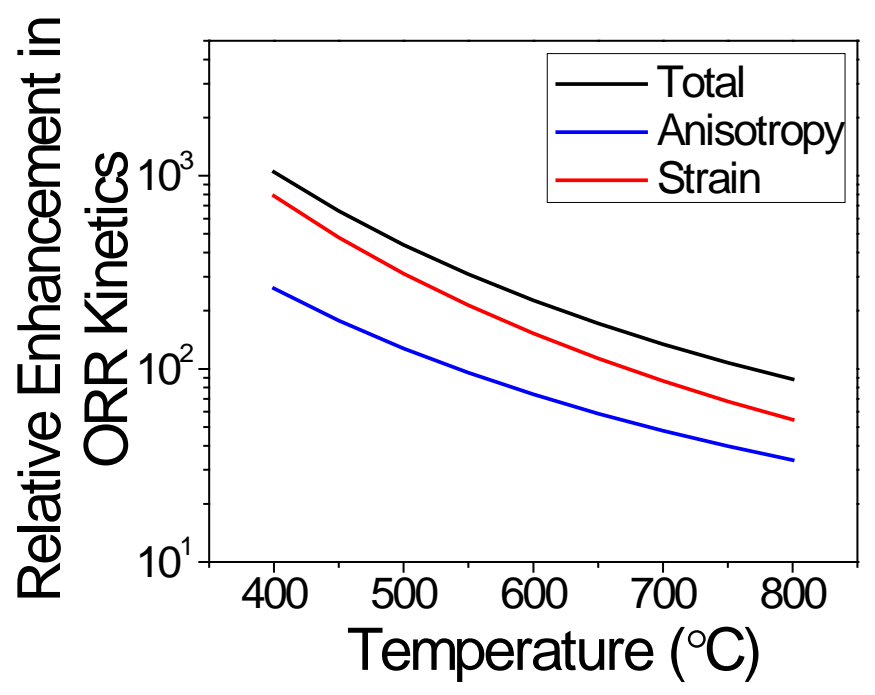

Figure 7. The relative enhancement of $\mathrm{ORR}$ at/near the $\mathrm{LSC}_{113} / \mathrm{LSC}_{214}$ interface, quantified as $\frac{k_{113 / 214}^{*}}{k_{113}^{*}}$ as a function of temperature. The deconvoluted contributions of the $\mathrm{LSC}_{214}$ anisotropy and the lattice strain in $\mathrm{LSC}_{113}$ and $\mathrm{LSC}_{214}$ (together) are also shown.

\section{Conclusion}

Using DFT $+U$ calculations, we demonstrated that, at the $\mathrm{LSC}_{113} / \mathrm{LSC}_{214}$ interface, the $\operatorname{LSC}_{214}(100)$ surface serves as an energetically favorable window for oxygen adsorption and incorporation. In addition to this, the lattice strain near the hetero-interface facilitates the oxygen incorporation especially on $\mathrm{LSC}_{113}$. These two factors together accelerate the ORR kinetics by $4 \times 10^{2}$ times at $500^{\circ} \mathrm{C}$. This finding explains to a large extent the experimental results of refs. 15 and 16 at $500-550^{\circ} \mathrm{C}$, and predict the RP phase anisotropy and the strain effects as two equally important sources of ORR activity enhancement reported for the $\mathrm{LSC}_{113} / \mathrm{LSC}_{214}$ hetero-structure. These results, however, quantitatively underestimate the $\times 10^{3}-10^{4}$ of enhancement reported experimentally. Both computational and experimental uncertainties may contribute to this difference. However, we believe that an important reason of this difference is the assessment of the single phase $\mathrm{LSC}_{113}$ and $\mathrm{LSC}_{214}$ separately in this work rather than an explicit representation of this $\mathrm{LSC}_{113} / \mathrm{LSC}_{214}$ interface. Recent experimental work in our group has shown that $\mathrm{LSC}_{214}$ is electronically activated when it interfaces the $\mathrm{LSC}_{113}$, and this electronic activation is expected to facilitate charge transfer from the surface to the oxygen adsorbates in the reduction process. ${ }^{35}$ 
This effect cannot be captured in our current DFT models. Nevertheless, such electronic activation of $\mathrm{LSC}_{214}$, concurrent with the anisotropically high oxygen incorporation into $\mathrm{LSC}_{214}$, is thought to be another key mechanism that additionally contributes to the fast ORR near the $\mathrm{LSC}_{113} / \mathrm{LSC}_{214}$ interface. Besides these sources, we cannot ignore that other factors such as space charge effects ${ }^{61}$ and a change in the electronic structure ${ }^{51,62}$ near the hetero-interface may also contribute to the ORR enhancement. Insights gained from our results can guide the design of novel perovskite/Ruddlesden-Popper composites for faster ORR kinetics. Hetero-structures with a large area of the (100) surfaces and smaller thickness in the [001] direction of the RP phase, and with tensile strain fields are expected to provide faster ORR kinetics for high-performance SOFC cathodes.

\section{Acknowledgement}

Authors acknowledge the US-DOE - Basic Energy Sciences, Grant No.DE-SC0002633 for financial support, and the National Science Foundation for computational support through the TeraGrid Advanced Support Program, Grant No.TG-ASC090058, and thank Sidney Yip and Harry Tuller at MIT for fruitful discussions on this topic.

\section{References}

1 B. C. H. Steele and A. Heinzel, Nature, 2001, 414, 345.

2 S. B. Adler, Chem. Rev., 2004, 104, 4791.

3 A. J. Jacobson, Chem. Mater., 2010, 22, 660.

4 L. Malavasi, C. A. J. Fisher and M. S. Islam, Chem. Soc. Rev., 2010, 39, 4370.

5 A. Chroneos, B. Yildiz, A. Tarancon, D. Parfitt and J. A. Kilner, Energy Environ. Sci., 2011, 4, 2774.

6 A. Tarancon, M. Burriel, J. Santiso, S. J. Skinner and J. A. Kilner, J. Mater. Chem., 2010, 20, 3799.

$7 \quad$ R. A. De Souza, M. Saiful Islam and E. Ivers-Tiffee, J. Mater. Chem., 1999, 9, 1621.

8 M. Pavone, A. M. Ritzmann and E. A. Carter, Energy Environ. Sci., 2011, 4, 4933.

9 M. E. Lynch, L. Yang, W. Qin, J.-J. Choi, M. Liu, K. Blinn and M. Liu, Energy Environ. Sci., 2011, 4, 2249.

10 S. P. Harvey, R. A. De Souza and M. Martin, Energy Environ. Sci., 2012.

11 M. S. D. Read, M. S. Islam, G. W. Watson and F. E. Hancock, J. Mater. Chem., 2001, 11, 2597.

12 A. Chroneos, D. Parfitt, J. A. Kilner and R. W. Grimes, J. Mater. Chem., 2010, 20, 266.

13 A. Kushima, D. Parfitt, A. Chroneos, B. Yildiz, J. A. Kilner and R. W. Grimes, Phys. Chem. Chem. Phys., 2011, 13, 2242.

14 A. B. Muñoz-García, M. Pavone and E. A. Carter, Chem. Mater., 2011, 23, 4525. 
15 M. Sase, K. Yashiro, K. Sato, J. Mizusaki, T. Kawada, N. Sakai, K. Yamaji, T. Horita and H. Yokokawa, Solid State Ionics, 2008, 178, 1843.

16 E. J. Crumlin, E. Mutoro, S.-J. Ahn, G. J. la O’, D. N. Leonard, A. Borisevich, M. D. Biegalski, H. M. Christen and Y. Shao-Horn, J. Phys. Chem. Lett., 2010, 1, 3149.

17 E. J. Opila, H. L. Tuller, B. J. Wuensch and J. Maier, J. Am. Ceram. Soc., 1993, 76, 2363.

18 L. Minervini, R. W. Grimes, J. A. Kilner and K. E. Sickafus, J. Mater. Chem., 2000, 10, 2349.

19 J. M. Bassat, P. Odier, A. Villesuzanne, C. Marin and M. Pouchard, Solid State Ionics, 2004, 167, 341.

20 C. N. Munnings, S. J. Skinner, G. Amow, P. S. Whitfield and I. J. Davidson, Solid State Ionics, 2005, 176, 1895.

21 M. Burriel, G. Garcia, J. Santiso, J. A. Kilner, R. J. Chater and S. J. Skinner, J. Mater. Chem., 2008, 18, 416.

22 S. Savvin, G. Mazo and A. Ivanov-Schitz, Crystallogr. Rep., 2008, 53, 291.

23 M. Yashima, M. Enoki, T. Wakita, R. Ali, Y. Matsushita, F. Izumi and T. Ishihara, J. Am. Chem. Soc., 2008, 130, 2762.

24 N. Schichtel, C. Korte, D. Hesse and J. Janek, Phys. Chem. Chem. Phys., 2009, 11, 3043. A. Kushima and B. Yildiz, J. Mater. Chem., 2010, 20, 4809. Z.-W. Wang, D.-J. Shu, M. Wang and N.-B. Ming, Phys. Rev. B, 2010, 82, 165309. J. W. Han and B. Yildiz, J. Mater. Chem., 2011, 21, 18983. R. A. De Souza, A. Ramadan and S. Horner, Energy Environ. Sci., 2012, 5, 5445. D.-J. Shu, S.-T. Ge, M. Wang and N.-B. Ming, Phys. Rev. Lett., 2008, 101, 116102. A. Kushima, S. Yip and B. Yildiz, Phys. Rev. B, 2010, 82, 115435. W. Donner, C. Chen, M. Liu, A. J. Jacobson, Y.-L. Lee, M. Gadre and D. Morgan, Chem. Mater., 2011, 23, 984.

32 H. Jalili, J. W. Han, Y. Kuru, Z. Cai and B. Yildiz, J. Phys. Chem. Lett., 2011, 2, 801. Z. Cai, Y. Kuru, J. W. Han, Y. Chen and B. Yildiz, J. Am. Chem. Soc., 2011, 133, 17696. M. J. Gadre, Y.-L. Lee and D. Morgan, Phys. Chem. Chem. Phys., 2012, 14, 2606. Y. Chen, Z. Cai, Y. Kuru, H. L. Tuller and B. Yildiz, in preparation, 2012. T. Nitadori, M. Muramatsu and M. Misono, Chem. Mater., 1989, 1, 215. V. V. Vashook, H. Ullmann, O. P. Olshevskaya, V. P. Kulik, V. E. Lukashevich and L. V. Kokhanovskij, Solid State Ionics, 2000, 138, 99.

39 J. P. Perdew, J. A. Chevary, S. H. Vosko, K. A. Jackson, M. R. Pederson, D. J. Singh and C. Fiolhais, Phys. Rev. B, 1992, 46, 6671.

$40 \quad$ P. E. Blöchl, Phys. Rev. B, 1994, 50, 17953.

41 S. L. Dudarev, G. A. Botton, S. Y. Savrasov, C. J. Humphreys and A. P. Sutton, Phys. Rev. B, 1998, 57, 1505.

42 S. J. Skinner and G. Amow, J. Solid State Chem., 2007, 180, 1977.

43 L. Bengtsson, Phys. Rev. B, 1999, 59, 12301.

44 J. Neugebauer and M. Scheffler, Phys. Rev. B, 1992, 46, 16067.

47 E. N. Armstrong, K. L. Duncan, D. J. Oh, J. F. Weaver and E. D. Wachsman, J. Electrochem. Soc., 2011, 158, B492. 
48 Y. A. Mastrikov, R. Merkle, E. Heifets, E. A. Kotomin and J. Maier, J. Phys. Chem. C, 2010, 114, 3017.

49 Y. Choi, D. S. Mebane, M. C. Lin and M. L. Liu, Chem. Mater., 2007, 19, 1690.

50 Y.-L. Lee, J. Kleis, J. Rossmeisl and D. Morgan, Phys. Rev. B, 2009, 80, 224101.

51 R. A. Evarestov, E. A. Kotomin, Y. A. Mastrikov, D. Gryaznov, E. Heifets and J. Maier, Phys. Rev. B, 2005, 72, 214411.

52 T. R. S. Prasanna and A. Navrotsky, J. Solid State Chem., 1994, 112, 192.

53 S. L. Stoll, A. M. Stacy and C. C. Torardi, Inorg. Chem., 1994, 33, 2761.

54 K. Toda, Y. Kameo, S. Kurita and M. Sato, J. Alloys. Compd., 1996, 234, 19.

$55 \quad$ S.-H. Byeon, K. Park and M. Itoh, J. Solid State Chem., 1996, 121, 430.

56 P. D. Battle, M. A. Green, N. S. Laskey, J. E. Millburn, L. Murphy, M. J. Rosseinsky, S. P. Sullivan and J. F. Vente, Chem. Mater., 1997, 9, 552.

57 J. Sloan, P. D. Battle, M. A. Green, M. J. Rosseinsky and J. F. Vente, J. Solid State Chem., 1998, 138, 135.

58 R. E. Schaak and T. E. Mallouk, J. Solid State Chem., 2001, 161, 225.

59 J. A. Rodgers, P. D. Battle, N. Dupré, C. P. Grey and J. Sloan, Chem. Mater., 2004, 16, 4257.

60 M. Sánchez-Andújar and M. A. Señarís-Rodríguez, Z. Anorg. Allg. Chem., 2007, 633, 1890.

61 X. Guo and J. Maier, Adv. Mater., 2009, 21, 2619.

62 J. Chakhalian, J. W. Freeland, H.-U. Habermeier, G. Cristiani, G. Khaliullin, M. van Veenendaal and B. Keimer, Science, 2007, 318, 1114. 